\title{
AUTOMORPHISM GROUPS OF COMPLEX DOUBLES OF KLEIN SURFACES
}

\author{
by E. BUJALANCE, $\dagger$ A. F. COSTA, $\ddagger$ G. GROMADZKI§ and D. SINGERMAN
}

(Received 22 January, 1993)

1. Introduction. In this paper we consider complex doubles of compact Klein surfaces that have large automorphism groups. It is known that a bordered Klein surface of algebraic genus $g \geq 2$ has at most $12(g-1)$ automorphisms. Surfaces for which this bound is sharp are said to have maximal symmetry. The complex double of such a surface $X$ is a compact Riemann surface $X^{+}$of genus $g$ and it is easy to see that if $G$ is the group of automorphisms of $X$ then $C_{2} \times G$ is a group of automorphisms of $X^{+}$. A natural question is whether $X^{+}$can have a group that strictly contains $C_{2} \times G$. In [8] C. L. May claimed the following interesting result: there is a unique Klein surface $X$ with maximal symmetry for which Aut $X^{+}$properly contains $C_{2} \times$ Aut $X$ (where Aut $X^{+}$denotes the group of conformal and anticonformal automorphisms of $X^{+}$).

Unfortunately there are errors in the proof and here we supply an alternative argument using inclusions between NEC groups. In particular, in Section 4, we prove a useful criterion on when we can extend an inclusion $\Gamma<\Delta$, with $\Delta$ a Fuchsian triangle group, to an inclusion between NEC groups. This enables us to show that there is one isomorphism class of groups that contains a group with signature $(2,2,2,3)$. By studying groups that contain $(2,2,2,4)$ we obtain a similar result concerning Klein surfaces that admit the second largest group of automorphisms, namely group of order $8(g-1)$. Finally some relations of the above results with the theory of regular maps on surfaces and real algebraic geometry are also pointed out.

2. Preliminaries. NEC groups. Let $\mathbb{C}^{+}$denote the upper half plane. With the Poincaré metric $d s=|d z| / y$, it becomes a model of hyperbolic plane. A non-Euclidean crystallographic (NEC) group is a discrete group $\Gamma$ of isometries of $\mathbb{C}^{+}$with respect to the hyperbolic metric and in this paper we shall assume that $\mathbb{C}^{+} / \Gamma$ is compact. If $\Gamma$ only contains orientation-preserving isometries then it is called a Fuchsian group; otherwise it is called a proper NEC group. Every proper NEC group contains a subgroup $\Gamma^{+}$of index 2 consisting of the elements of $\Gamma$ that preserve orientation. We call $\Gamma^{+}$the canonical Fuchsian group of $\Gamma$. An NEC group is determined algebraically by its signature

$$
\sigma(\Gamma)=\left(g ; \pm ;\left[m_{1}, \ldots, m_{r}\right] ;\left\{\left(n_{11}, \ldots, n_{1 s_{1}}\right), \ldots,\left(n_{k 1}, \ldots, n_{k s_{k}}\right)\right\}\right)
$$

If $\Gamma$ has this signature then $\mathbb{C}^{+} / \Gamma$ is an orbifold whose underlying space is a surface of genus $g$ with $k$ boundary components (holes) (see [10]). It is orientable if the + sign is

† Supported by DCICYT PB89-0201 and SCIENCE Program CEE ERB 4002 PL 910021.

$\ddagger$ Supported by DCICYT PB89-0201, SCIENCE Program CEE ERB 4002 PL 910021 and Acciones Hispano-Portuguesas.

$\S$ Supported by a grant of Spanish Ministery of Education.

Glasgow Math. J. 36 (1994) 313-330. 
used and nonorientable otherwise. There are $r$ cone points of angles $2 \pi / m_{1}, \ldots, 2 \pi / m_{r}$ in the interior of $\mathbb{C}^{+} / \Gamma$ and $s_{i}$ corner points of angle $\pi / n_{i 1}, \ldots, \pi / n_{i s_{i}}$ around the $i$ th hole. If $\Gamma$ has signature (2.1) then the area of a fundamental region for $\Gamma$ is $2 \pi \mu(\Gamma)$, where

$$
\mu(\Gamma)=\left(\alpha g+k-2+\sum_{i=1}^{r}\left(1-1 / m_{i}\right)+\sum_{i=1}^{k}\left(1-1 / n_{i j}\right) / 2\right),
$$

where

$$
\alpha=\left\{\begin{array}{l}
2 \text { if } \sigma(\Gamma) \text { has a }+ \text { sign } \\
1 \text { if } \sigma(\Gamma) \text { has a }- \text { sign }
\end{array}\right.
$$

(Alternatively, $-\mu(\Gamma)$ is the Euler characteristic of the orbifold). If $\Gamma_{1} \subseteq \Gamma$ then

$$
\left[\Gamma: \Gamma_{1}\right]=\mu\left(\Gamma_{1}\right) / \mu(\Gamma)
$$

A general presentation of $\Gamma$ of signature (2.1) can be written down ([3]). However in this paper we shall mainly be concerned with groups generated by reflections in the sides of a triangle or quadrilateral. A triangle group is one with signature $(0 ;+;[-] ;\{(k, l, m)\})$ and we denote it by $(k, l, m)$ for short. It has a presentation

$$
\left\langle c_{1}, c_{2}, c_{3}: c_{1}^{2}=c_{2}^{2}=c_{3}^{2}=\left(c_{1} c_{2}\right)^{k}=\left(c_{2} c_{3}\right)^{1}=\left(c_{1} c_{3}\right)^{m}=1\right\rangle .
$$

(In what follows we shall refer to any set of generating reflections satisfying the above relations to be a set of canonical generators for $\Gamma$ ). Its canonical Fuchsian group has signature $(0 ;+;[k, l, m] ;\{-\})$ A quadrilateral group has signature $(0 ;+;[-]$; $\{(k, l, m, n)\})$ which we shall abbreviate to $(k, l, m, n)$. It has a similar presentation to (2.4) with four reflection generators instead of three. In particular we shall be interested in the trirectangle groups $(2,2,2, n)$.

Klein surfaces. A Klein surface is a surface with a dianalytic structure $([\mathbf{1}],[3])$. In this paper all Klein surfaces will be compact and so homeomorphic to a surface with $g$ handles and $k$ holes or to a surface with $g$ cross-caps and $k$ holes. The algebraic genus is then $2 g+k-1$ if $X$ is orientable and $g+k-1$ if $X$ is nonorientable.

If $X$ is a Klein surface of algebraic genus $p \geq 2$ then there is an NEC group $\Gamma$ of signature $\left(g ; \pm ;[-] ;\left\{(-)^{k}\right\}\right)$ such that $X=\mathbb{C}^{+} / \Gamma$. Such groups are called surface groups or bordered surface groups if $k \geq 1$.

If $X$ is an orientable Klein surface without boundary then $X$ can be thought of as a Riemann surface. If $X$ is nonorientable or has boundary then we can form its complex double $X^{+}$which is a Riemann surface that admits an anticonformal involution $\tau$ such that $X \cong X^{+} /\langle\tau\rangle\left(X^{+}\right.$is unique up to conformal equivalence). An automorphism of $X$ can be lifted to an automorphism of $X^{+}$that commutes with $\tau$ and in this way we see that Aut $X$ is isomorphic to the centralizer of $\tau$ in $\operatorname{Aut}\left(X^{+}\right)$and then as in [8] that Aut $X^{+}$ contains a subgroup isomorphic to $C_{2} \times$ Aut $X$.

Automorphism groups of Klein surfaces. If $X$ is a Klein surface with boundary of algebraic genus $p \geq 2$ then it is known that $\mid$ Aut $X \mid \leq 12(p-1)$. When this bound is attained we say that $X$ has maximal symmetry. If $X=\mathbb{C}^{+} / \Gamma, \Gamma$ is a bordered surface group then this occurs if and only if $\Gamma$ is a normal subgroup of $(2,2,2,3)$. If this bound is not attained then $\mid$ Aut $X \mid \leq 8(p-1)$. 
3. Geometric comments. Some of the simplest examples of Klein surfaces with maximal symmetry are those that are homeomorphic to sphere with three holes. Since Thurston notes [10] such surfaces are called pair of pants and are formed by gluing together two isometric right-angled hexagons with identification as follows:

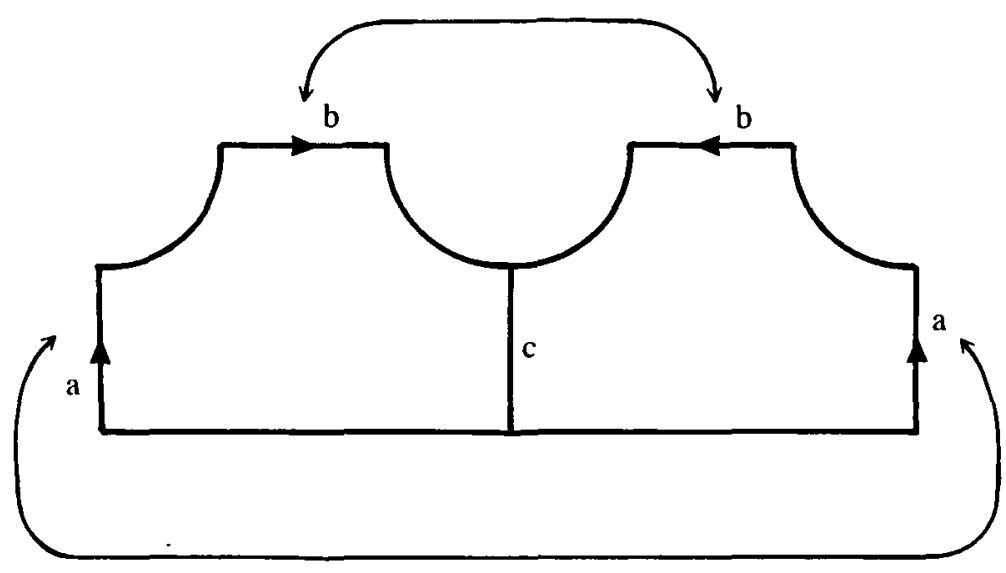

Figure 1.

This gives us a pair of pants $P$ with boundary components of hyperbolic length $2|\alpha|, 2|\beta|$, $2|\gamma|$, where || represents the hyperbolic length of an arc. Let us call $P$ equibordered if $2|\alpha|=2|\beta|=2|\gamma|$. Then clearly $P$ admits a group of automorphisms isomorphic to $C_{2} x D_{3}$ if and only if $P$ is equibordered. These are precisely the pairs of pants with maximal symmetry. The lenghts $|\alpha|,|\beta|,|\gamma|$ gives coordinates in the real Teichmüller space $\mathbf{T}(P)$ of $P$ so that the equibordered pants form a one-dimensional subspace of $\mathbf{T}(P)$.

Let us call a hyperbolic quadrilateral with angles $\pi / 2, \pi / 2, \pi / 2$ and $\alpha$, a trirectangle of angle $\alpha$. By [2, p. 161] we see that if $|\alpha|=|\beta|=|\gamma|$ then the other three sides of the hexagon have equal length and these are precisely the hexagons that can be decomposed into 6 isometric trirectangles of angle $\pi / 3$ as follows:

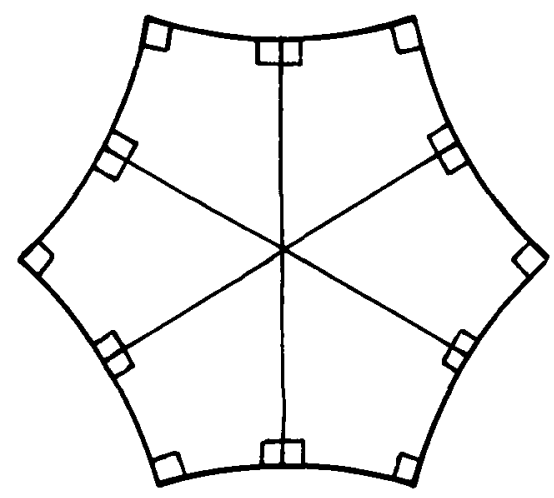

Figure 2. 
As the group of reflections in the sides of trirectangle of angle $\pi / 3$ is an NEC group of signature $(2,2,2,3)$ this corresponds to the inclusion $N<\Gamma$ with index 12 , where $N$ is an NEC group that uniformizes an equibordered pair of pants (i.e. one with maximal symmetry).

We now consider the special case, where the two sides of the trirectangle opposite to the angle $\pi / 3$ have equal length. Then by [2,p. 157] the other two sides have equal length. If this is the case then the above NEC group $\Gamma$ is a subgroup of index two in an NEC triangle group $\Delta=(2,4,6)$ as illustrated below.

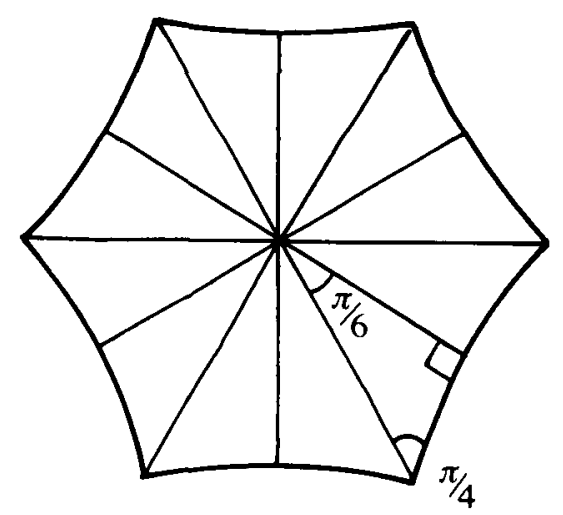

Figure 3.

As we shall prove in Theorem 3.4 this gives the only case of an NEC group properly containing a group of signature $(2,2,2,3)$. We then want to find NEC groups $M$ with $M \unlhd \Gamma$ and $M^{+} \unlhd \Delta$. This will lead to the result of May mentioned at the beginning.

If the two sides of the trirectangle opposite the angle $\pi / 3$ are equal then the corresponding hexagon is equilateral; all its sides have equal length. There is only one such right-angled hexagon; all sides can be calculated to have length equal to $\cosh ^{-1} 2$, by [2, p. 163]. Hence there is a unique pair of pants $P_{0}$ obtained by gluing together two such hexagons as in Figure 1. This pair of pants is not only equibordered but the joins $a, b$ and $\mathrm{c}$ have half the length of the boundaries. We call $P_{0}$ the regular pair of pants. Our main result (Theorem $\mathrm{A}$ ) is that of all Klein surfaces with maximal symmetry only $P_{0}$ has the property that Aut $P_{0}^{+}$has automorphism group strictly bigger than $C_{2} x$ Aut $P_{0}$. In fact we show that $\mid$ Aut $P_{0}^{c}|=48=4|$ Aut $P_{0} \mid$.

There is an interesting connection with maps on surfaces. As we are dealing with a normal subgroup of $(2,4,6)$ we expect to obtain a reflexible map by [5, chap. 8], or [7]. $P_{0}$ is composed of two hexagons so that $P_{0}^{+}$is composed of four hexagons. It is not difficult to 
show that the picture we get is as follows:

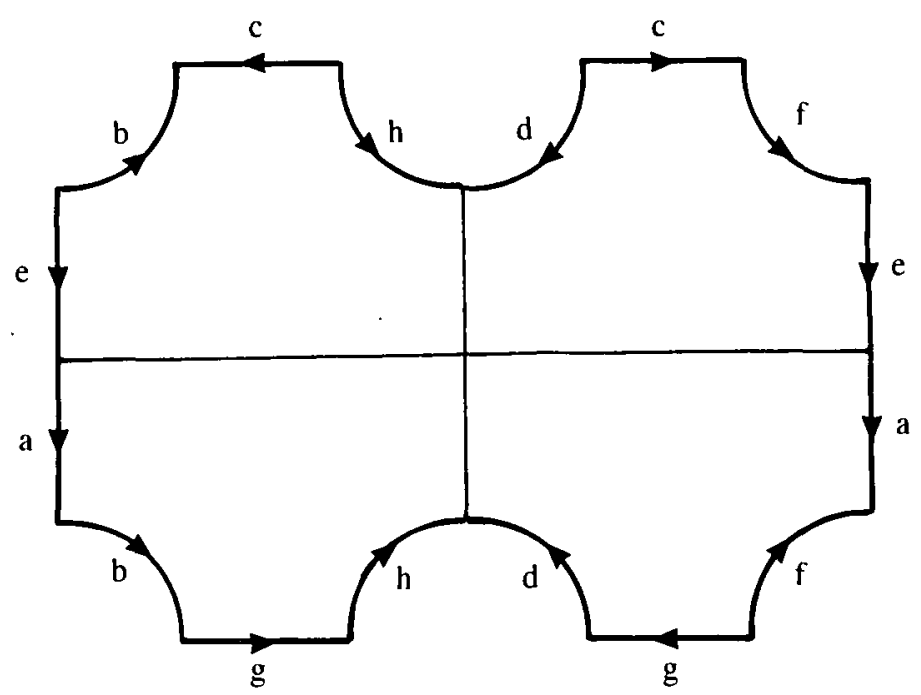

Figure 4 .

where we identify equally labelled edges orientably. We see that this is the same as the map of type $\{4,6\}$ in [5, Fig. 8.5] with 24 conformal automorphisms and hence with 48 automorphisms including those that reverse orientation. The surface $P_{0}^{+}$is called the Accola-Maclachlan surface of genus 2 (see [9]). It is thus obtained as the hyperelliptic surface branched over the six points of the unit circle and so corresponds to the algebraic curve of the equation $y^{2}=x^{6}-1 . P_{0}$ is then a Klein surface with maximal symmetry with the property that Aut $P_{0}^{+}$properly contains $C_{2} x$ Aut $P_{0}$. We shall prove that $P_{0}$ is the unique Klein surface with this property.

4. A combinatorial criterion for NEC extension of Fuchsian groups. Let $\Delta$ be a Fuchsian triangle group with signature $[k, l, m]$. Then $\Delta$ has presentation $\left\langle x, y: x^{k}, y^{\prime},(x y)^{m}\right\rangle$ and is contained in the NEC triangle group $\tilde{\Delta}$ with signature $(k, l, m)$ having the presentation (2.4).

We have $\tilde{\Delta}^{+}=\Delta$ and $x=c_{1} c_{2}, y=c_{2} c_{3}$. Then $c_{2} x c_{2}=x^{-1}$ and $c_{2} y c_{2}=y^{-1}$ and so we have an alternative presentation for $\tilde{\Delta}$

$$
\left\langle c_{2}, x, y: c_{2}^{2}=x^{k}=y^{\prime}=(x y)^{\prime \prime}=1, c_{2} x c_{2}=x^{-1}, c_{2} y c_{2}=y^{-1}\right\rangle .
$$

Let $\Gamma$ be a subgroup of $\Delta$ of index $N$. Then we can consider the Schreier coset graph $S(\Delta, \Gamma, x, y)$ whose vertices are right cosets $\Gamma g(g \in \Delta)$ and two vertices $\Gamma g$ and $\Gamma h$ are joined by a directed edge labelled by $x$ if $\Gamma g x=\Gamma h$ and by a directed edge labelled by $y$ if $\Gamma g y=\Gamma h$. If, $\Gamma g x^{2}=\Gamma g$ and if $\Gamma g x=\Gamma h$ then $\Gamma g$ and $\Gamma h$ are joined by a doubly directed edge $\leftrightarrow$. In practice, we usually describe the subgroup $\Gamma$ of $\Delta$ by the permutation representation i.e. by its Schreier coset graph. 
EXAMPLE. Let $\Delta$ be a triangle group $[2,8,3]=\left\langle x, y: x^{2}=y^{8}=(x y)^{3}\right\rangle$ and consider the homomorphism $\theta: \Delta \rightarrow S_{6}$ induced by the assignment

$$
x \mapsto(1,5)(3,6)(2)(4), \quad y \mapsto(1,2,3,4)(5,6) .
$$

So $x y \mapsto(1,6,4)(2,3,5)$. By [6] the subgroup $\Gamma=\theta^{-1}(\operatorname{Stab}(1))$ has signature $[2,2,2,4]$. The Schreier coset graph $S(\Delta, \Gamma, x, y)$ is as follows.

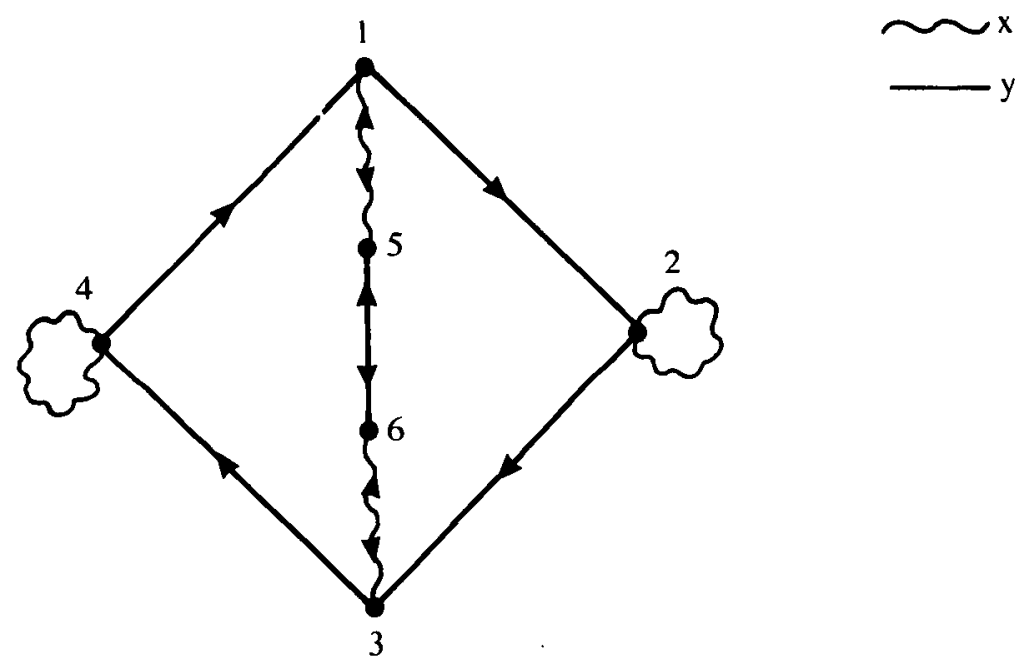

Figure 5.

In general we have a homomorphism $\theta: x \mapsto X \in S_{N}, y \mapsto Y \in S_{N}$, where $X$ and $Y$ are the permutations obtained by right multiplication of the cosets.

We now consider automorphisms of the coset graph; that is bijections of the graph preserving incidence and edge labelling. Each such automorphism $A$ permutes the vertices and so can be considered as a permutation in $S_{N}$.

Definition. A reflection $R$ of the coset graph $S(\Delta, \Gamma, x, y)$ is an automorphism of $S(\Delta, \Gamma, x, y)$ of order 2 that reverses the orientation of the edges. We then have $R X R^{-1}=X^{-1}, R Y R^{-1}=Y^{-1}$.

Remarks. (i) If the permutations $X, Y$ are of order 2 or 1 then the identity is regarded as a reflection.

(ii) Reflections maps cycles of $X$ (respectively $Y$ ) to cycles of $X$ (respectively $Y$ ) in the reverse order. In particular fixed points of $X$ (respectively $Y$ ) are mapped to fixed points of $X$ (respectively $Y$ ). This remark is useful too in showing that a reflection does not exist.

EXAMPLE. The above graph admits two reflections corresponding to geometric reflections in the horizontal and vertical axes:
(a) $(1,3)(5,6)(2)(4)$,
(b) $(1)(5)(6)(3)(2,4)$.

With the above notations we have the main result of this section.

THEOREM 4.1. Let $\Delta$ be a Fuchsian triangle group containing the group $\Gamma$ as a 
subgroup of index $N$. Then there are proper NEC-groups $\tilde{\Gamma}<\tilde{\Delta}$ for which $\Gamma$ and $\Delta$ are canonical Fuchsian subgroups of $\tilde{\Gamma}$ and $\tilde{\Delta}$ respectively if and only if $S(\Delta, \Gamma, x, y)$ admits a reflection.

Proof. Suppose that there is a proper NEC-group $\tilde{\Gamma}<\tilde{\Delta}$ with $\tilde{\Delta}^{+}=\Delta$ and $\tilde{\Gamma}^{+}=\Gamma$. Now if $\Delta=\bigcup_{i=1}^{N} \Gamma g_{i}$ is the decomposition of $\Delta$ into $\Gamma$-cosets then $\tilde{\Delta}=\bigcup_{i=1}^{N} \tilde{\Gamma} g_{i}$ since $g_{i} g_{j}^{-1} \in \Gamma$ if and only $g_{i} g_{j}^{-1} \in \tilde{\Gamma}$, as $g_{i}$ and $g_{j}$ preserve orientation. Thus if we consider the Schreier coset graph $S\left(\tilde{\Delta}, \tilde{\Gamma}, x, y, c_{2}\right)$ and we omit the edges corresponding to $c_{2}$ then we obtain a graph isomorphic to $S(\Delta, \Gamma, x, y)$. But the former graph admits an involution $R: \tilde{\Gamma} g \mapsto \tilde{\Gamma} g c_{2}$ and as $c_{2} x c_{2}=x^{-1}, c_{2} y c_{2}=y^{-1}, R$ is a reflection and thus $S(\Delta, \Gamma, x, y)$ admits a reflection.

For the converse, we suppose that $S(\Delta, \Gamma, x, y)$ admits a reflection $R$. We have a homomorphism $\theta: \Delta \rightarrow S_{N}$ defined by $\theta(x)=X, \theta(y)=Y$ as before. We can extend this to a homomorphism $\tilde{\theta}: \bar{\Delta} \rightarrow S_{N}$ by defining $\tilde{\theta}(x)=X, \tilde{\theta}(y)=Y, \tilde{\theta}\left(c_{2}\right)=R$. Via these homomorphisms both $\tilde{\Delta}$ and $\Delta$ act on the set of cosets as transitive permutation groups of degree $N$. Furthermore the second action extends the first one in the sense that if $g \in \Delta$ then the effect of $g$ is the same in both actions. Now $\Gamma$ is the stabilizer of a point $\alpha$ say in the first action. If $\tilde{\Gamma}$ is the stabilizer of $\alpha$ in the second action then $\Gamma \leq \tilde{\Gamma}$. Now $[\Delta: \Gamma]=[\tilde{\Delta}: \tilde{\Gamma}]=N$ as we have transitive actions and so the index of $\Gamma$ in $\tilde{\Gamma}$ is 2 . We now show that $\tilde{\Gamma}$ is a proper NEC-group. If not then $\tilde{\Gamma}$ is a Fuchsian group so that $\tilde{\Gamma} \leq \Delta$, and therefore $\Gamma \leq \tilde{\Gamma} \leq \Delta \leq \tilde{\Delta}$. If $g \in \tilde{\Gamma} \backslash \Gamma$ then $g$ fixes $\alpha$ in the second action. As $g \in \Delta$ and the second action extends the first one, $g \in \Gamma$ which is the stabilizer of $\alpha$ in the first action. This is a contradiction and therefore $\tilde{\Gamma}$ is a proper NEC group and $\tilde{\Gamma}^{+}=\Gamma$.

We now look for NEC groups that contain $(2,2,2,3)$. So we first look for Fuchsian groups that contain $[2,2,2,3]$ and apply the above theorem.

LEMMA 4.2. The only Fuchsian groups that contain $\Lambda=[2,2,2,3]$ are

$$
[2,3,7],[2,3,8],[2,3,9],[2,4,6] \text {. }
$$

Proof. As $\mu(\Lambda)=1 / 6$ any group $\Lambda^{*}$ containing $\Lambda$ as a subgroup of index $k$ has $\mu\left(\Delta^{*}\right)=1 /(6 k)$. This implies that either $\Lambda^{*}$ has one of the four signatures above or has signature $[2,3,12]$, or $[3,3,4]$. In the last two cases $k=2$. However the last group does not contain subgroup of index 2 as its abelianization is $C_{3}$, whilst using the permutation technique [6] any subgroup of index 2 in $[2,3,12]$ has a period 6 . Thus the only possibilities are those that are in the statement of the lemma.

The fact that they indeed do occur as groups containing $[2,2,2,3]$ can be proved by exhibiting the permutation representations and using [6]. In each case it is easy to show that the corresponding representation is essentially unique, apart from relabelling the symbols. For the next theorem we also give here the corresponding Schreier coset graphs.

To see when a Fuchsian inclusion can be extended to an NEC inclusion we use Theorem 4.1. This implies that in case (i) no such extension is possible. In cases (ii) and (iii) one such extension is possible and in case (iv) two such extensions are possible as the Schreier coset diagram admits two essentially distinct reflections; one in the vertical and one in the horizontal axes. To distinguish between the two possible NEC extensions we use a lemma which tells us when the NEC extension has a finite order element that can not be written as product of reflections of the group.

Let $\Delta=\left\langle x, y: x^{2}, y^{\prime},(x y)^{m}\right\rangle$ be a Fuchsian triangle group containing a group $\Gamma$ as a 
(i)

$$
\begin{aligned}
& \Lambda^{*}=[2,3,7] \\
& x \mapsto(2,3)(4,7)(1)(5)(6), \\
& y \mapsto(1,2,4)(5,6,7)(3), \\
& x y \mapsto(1,2,3,4,5,6,7) .
\end{aligned}
$$

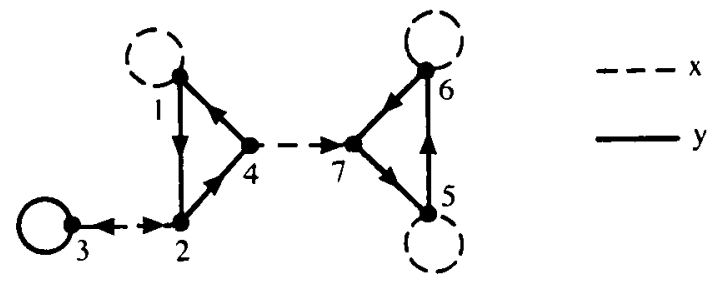

(ii) $\quad \Lambda^{*}=[2,3,8]$

$x \mapsto(1,2)(3)(4)$,

$y \mapsto(1,3,4)(2)$,

$x y \mapsto(1,2,3,4)$.

(iii) $\quad \Lambda^{*}=[2,3,9]$

$x \mapsto(1)(2)(3)$,

$y \rightarrow(1,2,3)$,

$x y \mapsto(1,2,3)$.

(iv) $\quad \Lambda^{*}=[2,4,6]$

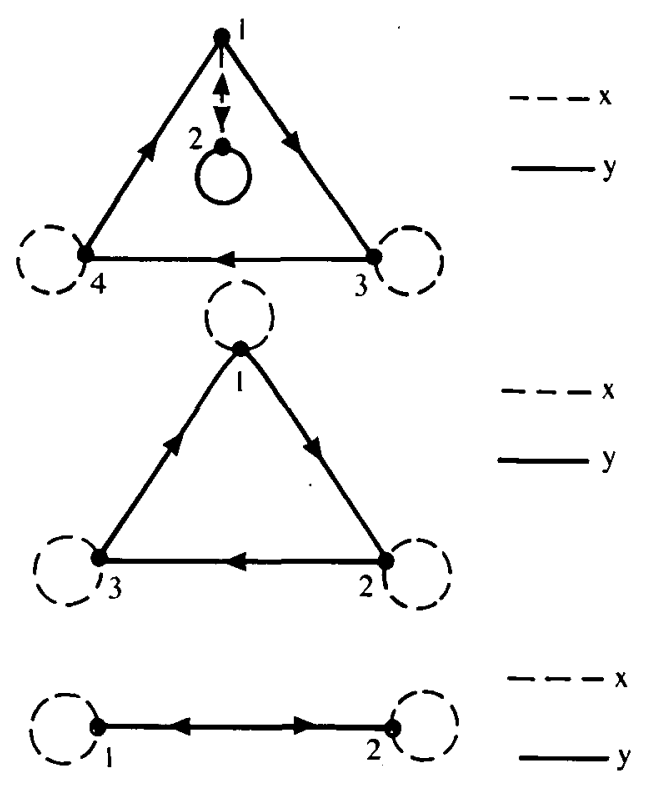

$x \mapsto(1)(2)$

$y \mapsto(1,2)$

$x y \mapsto(1,2)$

Figure 6.

subgroup of index $N$. Assume that $S(\Delta, \Gamma, x, y)$ admits a reflection $R$. Let $\tilde{\Gamma}$ and $\tilde{\Delta}$ be the proper NEC groups given by Theorem 4.1.

Leмma 4.3. If the reflection interchanges two loops with label $x$ in $S(\Delta, \Gamma, x, y)$ then the group $\tilde{\Gamma}$ has an order two element which is not a product of two reflections of $\tilde{\Gamma}$.

Proof. This follows directly from Theorem 4.5 of [4]. It is also a very special case of a Theorem of Hoare [6].

THEOREM 4.4. The only NEC group that contains an NEC group $\Lambda$ with signature $(2,2,2,3)$ is a group $\Lambda^{*}$ with signature $(2,4,6)$.

Proof. We consider the Schreier coset diagrams in Lemma 4.2. (i) admits no reflection and so $(2,2,2,3)$ is not a subgroup of $(2,3,7)$ by Theorem 4.1. Coset diagrams in (ii) and (iii) in Lemma 4.2 do admit reflections and so $(2,3,8)$ and $(2,3,9)$ do contain an NEC group whose canonical Fuchsian group is $[2,2,2,3]$. These reflections interchange loops with label $x$ so by Lemma 4.3 these NEC groups contain an elliptic element that is not the product of two reflections. The NEC groups with canonical Fuchsian group $[2,2,2,3]$ have signatures $(2,2,2,3)$ or $(0 ;+,[2],\{(2,3)\})$ and only the latter contain such elliptic elements. This gives the inclusion $(0,+,[2],\{(2,3)\})<(2,3, k)$ with $k=8$ or 9 . The diagram (iv) admits reflections on both horizontal and vertical axes. One reflection 
interchanges $x$ loops the other does not. Thus both $(0 ;+,[2],\{(2,3)\})$ and $(2,2,2,3)$ are contained in $(2,4,6)$. In particular $(2,4,6)$ is the only NEC group that contains $(2,2,2,3)$.

5. Groups of automorphisms of Riemann double covers of surfaces with maximal symmetry. We start with the following general result which is proved in [8].

Proposition 5.1. Let $G$ be a group of automorphisms of a bordered Klein surface $X$ of algebraic genus $p \geq 2$ and let $X^{+}$be the canonical Riemann double of $X$. Then $\operatorname{Aut}\left(X^{+}\right)$ contains a subgroup isomorphic to $C_{2} \times G$.

THEOREM A. Let $X$ be a bordered Klein surface with maximal symmetry of algebraic genus $p \geq 2$ that is different from the regular pair of pants. Then $\operatorname{Aut}\left(X^{+}\right)=C_{2} \times$ Aut $X$.

Proof. Let $X=\mathbb{C}^{+} / \Gamma$. Then $G=$ Aut $X=\Lambda / \Gamma$ for some NEC surface group $\Gamma$ and an NEC group $\Lambda$ with signature $(2,2,2,3)$. By Proposition $5.1, \Lambda / \Gamma^{+} \cong C_{2} \times G \subseteq \operatorname{Aut}\left(X^{+}\right)$; in particular $\left|\operatorname{Aut}\left(X^{+}\right)\right| \geq 24(p-1)$. Now if $\left|\operatorname{Aut}\left(X^{+}\right)\right|>24(p-1)$ then $\operatorname{Aut}\left(X^{+}\right)=\bar{\Lambda} / \Gamma^{+}$, where by Theorem $4.4 \tilde{\Lambda}$ has signature $(2,4,6)$. Denote by $\theta$ and by $\tilde{\theta}$ the canonical epimorphisms $\Lambda \rightarrow \Lambda / \Gamma$ and $\tilde{\Lambda} \rightarrow \tilde{\Lambda} / \Gamma^{+}$respectively.

Let $\tilde{c}_{0}, \tilde{c}_{1}$ and $\tilde{c}_{2}$ be the reflections in the sides of a triangle with angles $\pi / 2, \pi / 4, \pi / 6$ chosen so that $\left(\tilde{c}_{0} \tilde{c}_{1}\right)^{2}=\left(\tilde{c}_{1} \tilde{c}_{2}\right)^{4}=\left(\tilde{c}_{0} \tilde{c}_{0}\right)^{6}=1$. From Figure 7 we see that

$$
c_{0}=\tilde{c}_{2} \tilde{c}_{0} \tilde{c}_{2}, \quad c_{1}=\tilde{c}_{2} \tilde{c}_{1} \tilde{c}_{2}, \quad c_{2}=\tilde{c}_{1}, \quad c_{3}=\tilde{c}_{0}
$$

are the canonical reflection generators of $\Lambda$. We have $\left(c_{0} c_{1}\right)^{2}=\left(c_{1} c_{2}\right)^{2}=\left(c_{2} c_{3}\right)^{2}=$ $\left(c_{3} c_{0}\right)^{3}=1$.

As $\Gamma$ is a bordered surface group it must contain some of the reflection generators of $\Lambda$. If $c_{o} \in \Gamma$ then from the above relations $c_{3} \in \Gamma$ and so $c_{0} c_{3} \in \Gamma$ which is not true as $\Gamma$ is a bordered surface group. Thus $c_{0} \notin \Gamma$ and similarly $c_{3} \in \Gamma$. Therefore $c_{1} \in \Gamma$ or $c_{2} \in \Gamma$. In the first case $\left(c_{1} c_{3}\right)^{2}=c_{1}\left(c_{3} c_{1} c_{3}\right) \in \Gamma^{+}$and in the second case $\left(c_{0} c_{2}\right)^{2} \in \Gamma^{+}$. By (5.1) we find that in both cases

$$
\left(\tilde{c}_{2} \tilde{c}_{1} \tilde{c}_{2} \dot{\tilde{c}}_{0}\right)^{2} \in \Gamma^{+}
$$

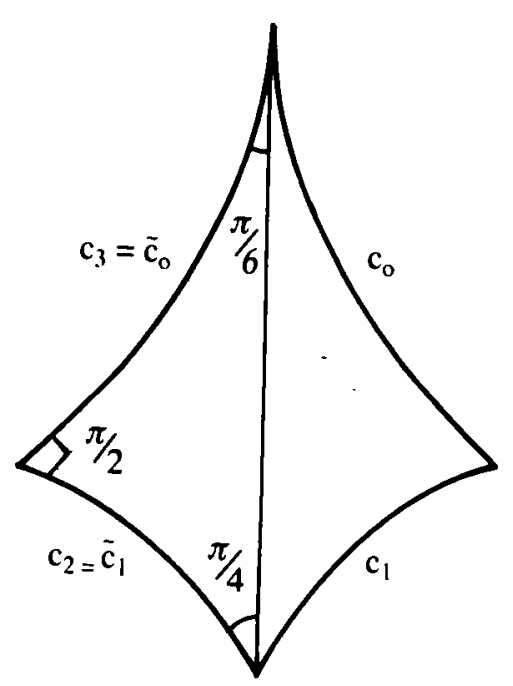

Figure 7. 
As $\Gamma^{+}$is a Fuchsian surface group we see that the images $x, y$ and $z$ of $\tilde{c}_{0}, \tilde{c}_{1}$ and $\tilde{c}_{2}$ are, in $\tilde{\Lambda} / \Gamma^{+}=\tilde{\mathrm{G}}$, elements of order 2 and $x y, y z$ and $x z$ have orders 2,4 and 6 respectively. Furthermore $(z y z x)^{2}=1$, by (5.2). Thus $\tilde{G}$ is a factor group of the group $F$ with presentation

$$
\left\langle x, y, z \mid x^{2}, y^{2}, z^{2},(x y)^{2},(y z)^{4},(x z)^{6},(z y z x)^{2}\right\rangle
$$

If $A=x y, B=y z$ then $F$ has a subgroup $F^{+}$of index 2 with presentation

$$
\left\langle A, B \mid A^{2}, B^{4},(A B)^{6},\left(B^{-2} A\right)^{2}\right\rangle
$$

The group generated by $B^{2}$ is central and $F^{+} /\left\langle B^{2}\right\rangle \simeq D_{6}$, Thus $F^{+}$has order 24 and $F$ has order 48.

On the other hand, by (2.3) $|\tilde{G}|$ is a multiple of 48 and therefore $\tilde{G}$ has the presentation (5.3). So $X$ has algebraic genus $p=2$. Finally observe that $\left(c_{i} c_{j}\right)^{\alpha} \in \Gamma$ if and only if $\left(c_{i} c_{j}\right)^{\alpha} \in \Gamma^{+}$and therefore, writing $k_{i j}=$ order of $\theta\left(c_{i j}\right)$, from [3, Theorem 2.3.3] and (5.2) that $\Gamma$ has $|G| / 2 k_{02}=12 / 4=3$ empty period cycles if $c_{1} \in \Gamma$ and $|G| / 2 k_{13}=12 / 4=3$ empty period cycles if $c_{2} \in \Gamma$ (this result also follows from [6]). In either case we see that $X=\mathbb{C}^{+} / \Gamma$ is a sphere with three holes as claimed.

The existence of this surface was proved in Section 3.

6. Groups of automorphisms of Riemann double covers of bordered Klein surfaces with group of automorphisms of order $8(p-1)$. Using the same techniques, as in the proof of Theorem 4.4 we prove the following result.

TheOREM 6.1. The groups $(2,4,8),(2,4,6),(2,4,5)$ and $(2,3,8)$ are the only NEC groups that contain an NEC group $\Lambda$ with signature $(2,2,2,4)$.

Proof. Indeed it is not difficult to see as in the proof of Lemma 4.2 that the only Fuchsian groups that contain $\Lambda^{+}=[2,2,2,4]$ are

$$
[2,4,8],[2,4,6],[2,3,12],[2,4,5],[2,3,8]
$$

and the corresponding Schreier graphs are
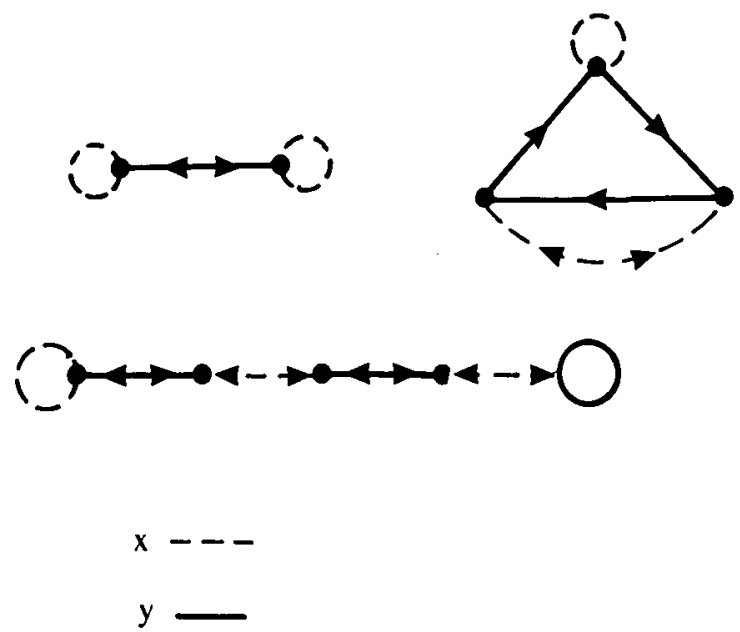
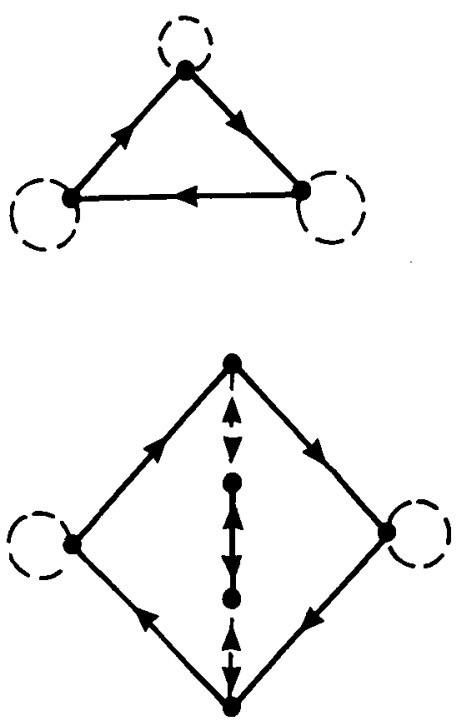
Now we see that the graphs admitting a reflection that do not interchange two loops correspond to groups $[2,4,8],[2,4,6],[2,4,5]$ and $[2,3,8]$. This completes the proof.

We are in the position to prove our second theorem.

THEOREM B. Let $X$ be a bordered Klein surface of algebraic genus $p \geq 2$ that has the group of automorphisms of order $8(p-1)$ and is different from four Klein surfaces of the following topological types:

(i) a projective plane with two holes,

(ii) a torus with one hole,

(iii) a torus with two holes,

(iv) a sphere with four holes and

(v) a torus with four holes. Then $\operatorname{Aut}\left(X^{+}\right)=C_{2} \times$ Aut $X$.

Proof. As in Theorem A, let us write $X=\mathbb{C}^{+} / \Gamma$ for some bordered surface group $\Gamma$ and $G=$ Aut $X=\Lambda / \Gamma$ for some NEC group $\Lambda$ with signature $(2,2,2,4)$. Let $\tilde{G}=$ Aut $\left(X^{+}\right)$and assume that $|\tilde{G}|>2|G|$. Then $\tilde{G}=\tilde{\Lambda} / \Gamma^{+}$, where by $4.4 .2, \tilde{\Lambda}$ has one of the following signatures:

(1) $(2,3,8)$ and $|\tilde{G}|=96(p-1)$,

(2) $(2,4,6)$ and $|\tilde{G}|=48(p-1)$,

(3) $(2,4,8)$ and $|\tilde{G}|=32(p-1)$.

(4) $(2,4,5)$ and $\mid \tilde{G} \|=80(p-1)$.

Let us denote by $\theta$ and $\tilde{\theta}$ the canonical epimorphisms $\Lambda \rightarrow \Lambda / \Gamma$ and $\tilde{\Lambda} \rightarrow \tilde{\Lambda} / \Gamma^{+}$ respectively.

Case (1). First of all we can have the inclusion $\Lambda<\tilde{\Lambda}$ where $\Lambda$ has signature $(2,2,2,4)$ and $\tilde{\Lambda}$ has signature $(2,3,8)$. This follows from the Example in Section 4 but more directly from the decomposition of the trirectangle with angles $\pi / 2, \pi / 2, \pi / 2, \pi / 4$ into 6 triangles with angles $\pi / 2, \pi / 3, \pi / 8$

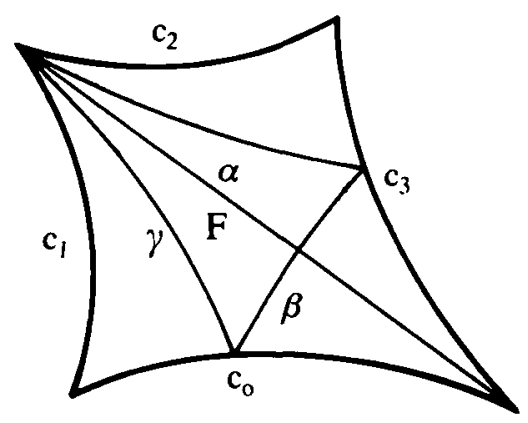

Figure 8.

The $(2,3,8)$ triangle $F$ has sides $\alpha, \beta, \gamma$. Let $\tilde{c}_{0}, \tilde{c}_{1}, \tilde{c}_{2}$ be reflections in the sides. Then

$$
\tilde{c}_{0}^{2}=\tilde{c}_{1}^{2}=\tilde{c}_{2}^{2}=\left(\tilde{c}_{0} \tilde{c}_{1}\right)^{2}=\left(\tilde{c}_{1} \tilde{c}_{2}\right)^{3}=\left(\tilde{c}_{0} \tilde{c}_{2}\right)^{8}=1
$$

As $\Lambda$ is generated by reflections in the sides of the quadrilateral in Figure 8 , it is generated by:

$$
c_{0}=\tilde{c}_{2} \tilde{c}_{1} \tilde{c}_{2}, \quad c_{1}=\tilde{c}_{2} \tilde{c}_{0} \tilde{c}_{2}, \quad c_{2}=\tilde{c}_{0} \tilde{c}_{2} \tilde{c}_{0} \tilde{c}_{2} \tilde{c}_{0}, \quad c_{3}=\tilde{c}_{0} \tilde{c}_{2} \tilde{c}_{1} \tilde{c}_{2} \tilde{c}_{0}
$$


Then $c_{0}^{2}=c_{1}^{2}=c_{2}^{2}=c_{3}^{2}=\left(c_{0} c_{1}\right)^{2}=\left(c_{1} c_{2}\right)^{2}=\left(c_{2} c_{3}\right)^{2}=\left(c_{3} c_{0}\right)^{4}=1$. Let $\Lambda^{*}$ be the triangle group generated by the reflections $\tilde{c}_{0}, \tilde{c}_{1}$ and $\tilde{c}_{2}$.

As $\Gamma$ is a bordered surface group then $c_{1}$ or $c_{2}$ belongs to $\Gamma$. Let $c_{1} \in \Gamma$, as $\Gamma$ is normal subgroup of $\Lambda$ then $c_{3} c_{1} c_{3} \in \Gamma$ so $\left(c_{1} c_{3}\right)^{2} \in \Gamma$ and hence $\left(c_{1} c_{3}\right)^{2} \in \Gamma^{+}$. Now $c_{1} c_{3}=\tilde{c}_{2}$ $\tilde{c}_{0} \tilde{c}_{2} \tilde{c}_{0} \tilde{c}_{2} \tilde{c}_{1} \tilde{c}_{2} \tilde{c}_{0}$. Let $\tilde{\theta}\left(\tilde{c}_{0}\right)=x, \theta\left(\tilde{c}_{1}\right)=y, \theta\left(\tilde{c}_{2}\right)=z$. Then $\tilde{\theta}\left(c_{1} c_{3}\right)=(z x)^{2} z y z x$. Let $A=x y$, $B=y z$. Then $A B=x z$. Now $A, B$ generate the subgroup $\tilde{\Lambda}^{+} / \Gamma^{+}$and $\theta\left(c_{1} c_{3}\right)=$ $(A B)^{-2} B^{-1}(A B)^{-1}$ so that $\left(A B^{2}(A B)^{2}\right)^{2}=1$. By conjugating by $A B$ we see this relation is equivalent to $\left(B(A B)^{3}\right)^{2}=1$ or $\left((A B)^{3} B\right)^{2}=1$. This is equivalent to $\left((A B)^{4} B(A B)^{-1}\right)^{2}=1$ or $\left((A B)^{4} A\right)^{2}=1$. Thus $\tilde{\Lambda}^{+} / \Gamma^{+}$obeys the relations $A^{2}=B^{3}=(A B)^{8}=\left((A B)^{4} A\right)^{2}=1$. This is a presentation of $\mathrm{GL}(2,3)$ or order 48 (see 8.8 of $[5]$ ).

Similarly if $c_{2} \in \Gamma$, then $c_{0} c_{2} c_{0} \in \Gamma$ and $\left(c_{0} c_{2}\right)^{2} \in \Gamma^{+}$. Therefore $\left(\tilde{c}_{2} \tilde{c}_{1} \tilde{c}_{2} \tilde{c}_{0} \tilde{c}_{2} \tilde{c}_{0} \tilde{c}_{2} \tilde{c}_{0}\right)^{2} \in$ $\Gamma^{+}$and we get the relation $\left(B^{-1}(A B)^{-3}\right)^{2}=1$ in $\tilde{\Lambda}^{+} / \Gamma^{+}$. As above this shows that $\tilde{\Lambda}^{+} / \Gamma^{+} \simeq \mathrm{GL}(2,3)$. Then the group $\tilde{\Lambda} / \Gamma^{+}=\tilde{G}$ is a $C_{2}$-extension of $\operatorname{GL}(2,3)$ and has 96 elements. By (2.3), $\Gamma$ has algebraic genus $p=2$ and by [3, Theorem 2.3.3] $\Gamma$ has $k=8 / 2 q$ empty period cycles, where $q$ is the order of the image of $c_{0} c_{2}$ in $\tilde{G}$ if $c_{1} \in \Gamma$ and the image of $c_{1} c_{3}$ in $\tilde{G}$ if $c_{2} \in \Gamma$. Thus in both cases $q=2$ and so using (2.2) we see that $\Gamma$ has signature $(1 ;-;[-] ;\{(-)(-)\})$ and therefore $X$ is a real projective plane with two boundary components (see also [4]).

Conversely the above arguments shows that this exceptional surface exists. Indeed take an NEC group $\tilde{\Lambda}$ with signature $(2,3,8)$, the group $\tilde{G}$ with the presentation

$$
\left\langle x, y, z \mid x^{2}, y^{2}, z^{2},(x y)^{2},(y z)^{3},(x z)^{8},\left((z x)^{2} z y z x\right)^{2}\right\rangle
$$

that by the above arguments have order 96 , and consider an epimorphism $\tilde{\theta}: \hat{\Lambda} \rightarrow \tilde{G}$ given by

$$
\tilde{\theta}\left(\tilde{c}_{0}\right)=x, \quad \tilde{\theta}\left(\tilde{c}_{1}\right)=y, \quad \tilde{\theta}\left(\tilde{c}_{2}\right)=z .
$$

Then it is straightforward to check, using results of Chapter 2 of [3], that for $\Gamma=\operatorname{Ker} \tilde{\theta} \cap \Lambda, X=\mathbb{C}^{+} / \Gamma$ is a surface we are looking for. The surface $X^{+}$is the underlying surface of a regular map of type $\{3,8\}$ on a surface of genus 2 , such a map is unique [5] and so the corresponding Riemann surface is unique [7]. As the reflections $c_{1}$, $c_{2}$ are conjugate they must give dianalytically equivalent Klein surfaces. In Figure 9 we have the map on $X^{+}$and the symmetry on the map giving as quotient the projective plane with two holes. We remark that the surface $X^{+}$is hyperelliptic (it has genus 2 ) and that the hyperelliptic involution is given by $\left(\tilde{c}_{0} \tilde{c}_{2}\right)^{4}$.

Case (2). Let $\tilde{c}_{0}, \tilde{c}_{1}, \tilde{c}_{2}$, be the reflections in the sides $\alpha, \beta, \gamma$ of the $(2,4,6)$ triangle $F$ in Figure 10. Then by the decomposition of the $(2,2,2,4)$ trirectangle in the Figure 10 we have:

$$
c_{0}=\tilde{c}_{1}, \quad c_{1}=\tilde{c}_{0} \tilde{c}_{2} \tilde{c}_{1} \tilde{c}_{2} \tilde{c}_{0}, \quad c_{2}=\tilde{c}_{0} \tilde{c}_{2} \tilde{c}_{0} \tilde{c}_{2} \tilde{c}_{0}, \quad c_{3}=\tilde{c}_{2} .
$$

Now since $\Gamma$ is a bordered surface group $c_{1} \in \Gamma$ or $c_{2} \in \Gamma$. But then $\left(c_{1} c_{3}\right)^{2} \in \Gamma^{+}$in the first case and $\left(c_{0} c_{2}\right)^{2} \in \Gamma^{+}$in the second one. Observe that $\left(c_{i} c_{j}\right)^{\alpha} \in \Gamma$ if and only if $\left(c_{i} c_{j}\right)^{\alpha} \in \Gamma^{+}$and

$$
\begin{aligned}
& \left(c_{1} c_{3}\right)^{2}=\left(\tilde{c}_{0} \tilde{c}_{2} \tilde{c}_{1} \tilde{c}_{2} \tilde{c}_{0} \tilde{c}_{2}\right)^{2}, \\
& \left(c_{0} c_{2}\right)^{2}=\left(\tilde{c}_{1} \tilde{c}_{0} \tilde{c}_{2} \tilde{c}_{0} \tilde{c}_{2} \tilde{c}_{0}\right)^{2}
\end{aligned}
$$



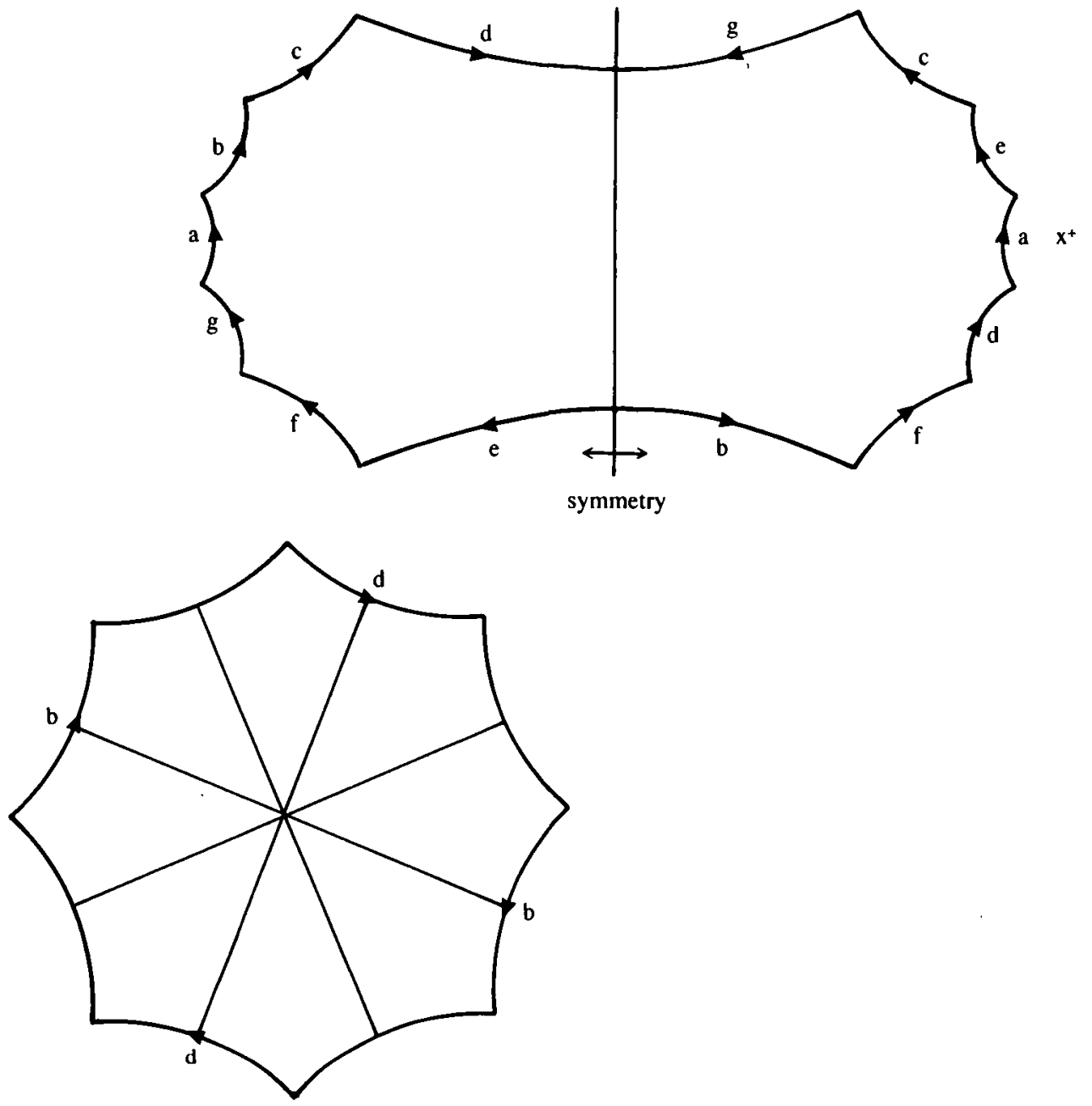

Figure 9.

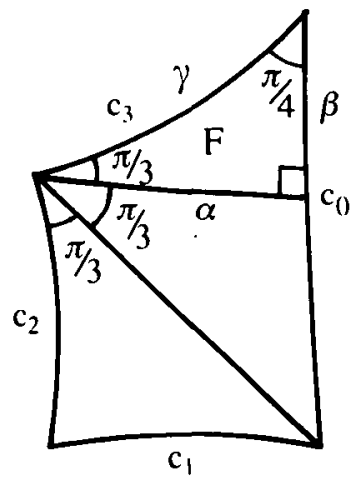

Figure 10. 
Consider the first case. Then, by exactly the same arguments as in the case (1),

$$
\tilde{\Lambda} / \Gamma^{+}=\tilde{G}=\left\langle x, y, z \mid z^{2}, y^{2}, z^{2},(x y)^{2},(y z)^{4},(x z)^{6},\left(y(x z)^{3}\right)^{2}\right\rangle .
$$

As $(x z)^{3}$ is central and $\tilde{G} /\left\langle(z x)^{3}\right\rangle$ has order $48, \tilde{G}$ has order 96 . So $X$ has algebraic genus $p=3$ by (2.3). Moreover $\tilde{\theta}\left(c_{0} c_{2}\right)=y z x z \in \Lambda / \Gamma^{+}$has order 4 . So, by [3, Theorem 2.3.3], $\Gamma$ has two empty period cycles. Finally $c_{2} c_{3}$ and $c_{0} c_{3}$ become in $\Lambda / \Gamma^{+}$two commuting elements of order 2 and 4 . Thus $X$ is orientable by [3, Theorem 2.1.3] and therefore it is a torus with two holes. Observe that the above surface is hyperelliptic and the hyperelliptic involution is induced by $\left(\tilde{c}_{0} \tilde{c}_{2}\right)^{3}$. The group $\tilde{G}$ is the symmetry group of the map of type $\{4,6\}$ on a surface of genus 3 shown in Figure 11. We also illustrate the symmetry of this map whose quotient is a torus with two holes.

In the second case

$$
\tilde{\Lambda} / \Gamma^{+}=\tilde{G}=\left\langle x, y, z \mid x^{2}, y^{2}, z^{2},(x y)^{2},(y z)^{4},(x z)^{6},\left[y,(x z)^{2}\right]\right\rangle .
$$

Now the above group has order 48 and again $\tilde{\theta}\left(c_{1} c_{3}\right)=x z y z x z$ has order 4 so that $X$ has one hole. This time $\tilde{\theta}\left(c_{1} c_{3}\right)=z y$ and $\tilde{\theta}\left(c_{0} c_{3}\right)=y z$ and thus $X$ is also orientable. Therefore $X$ is a torus with one hole that is hyperelliptic because it has genus 2 . As in the
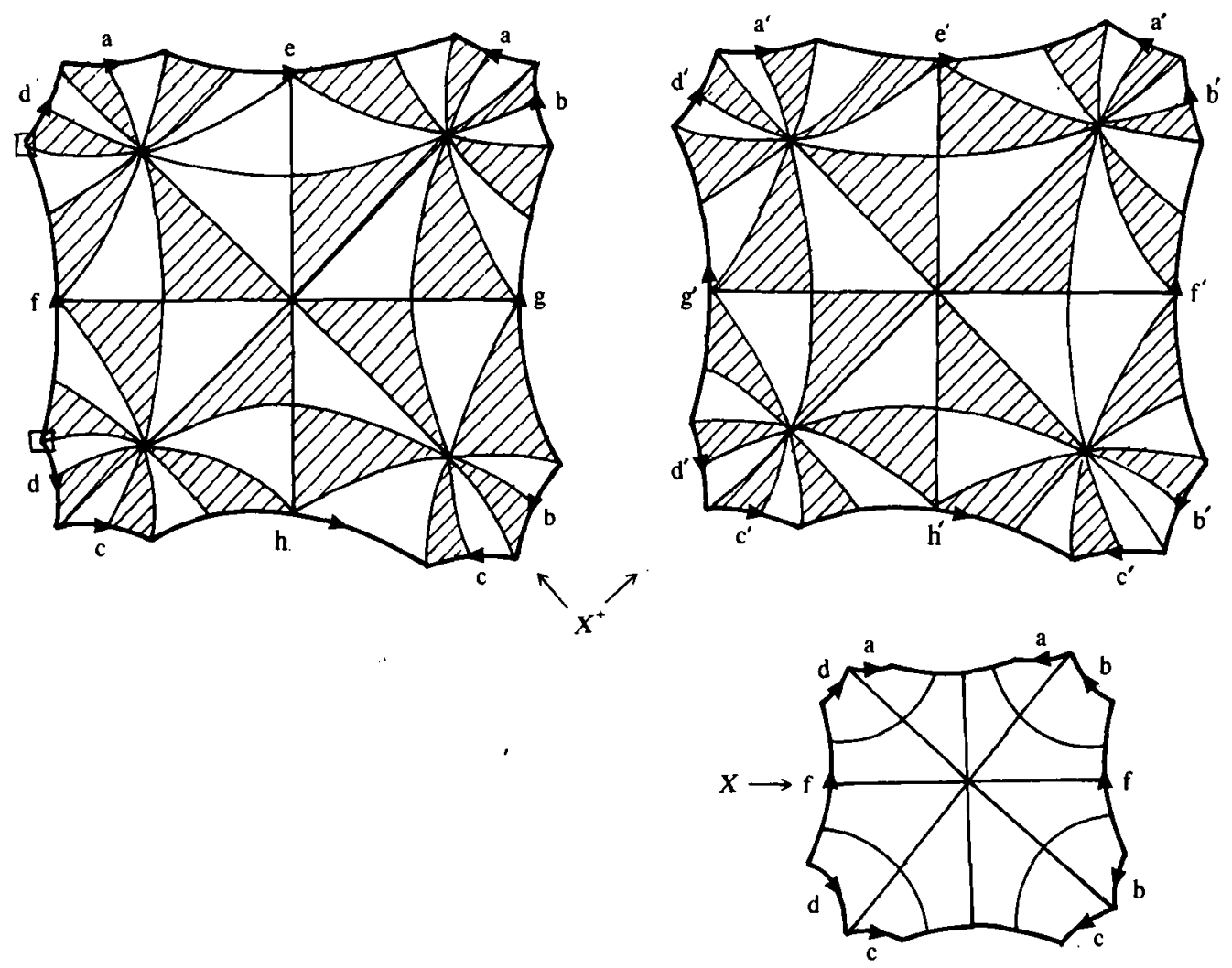

Figure 11. 

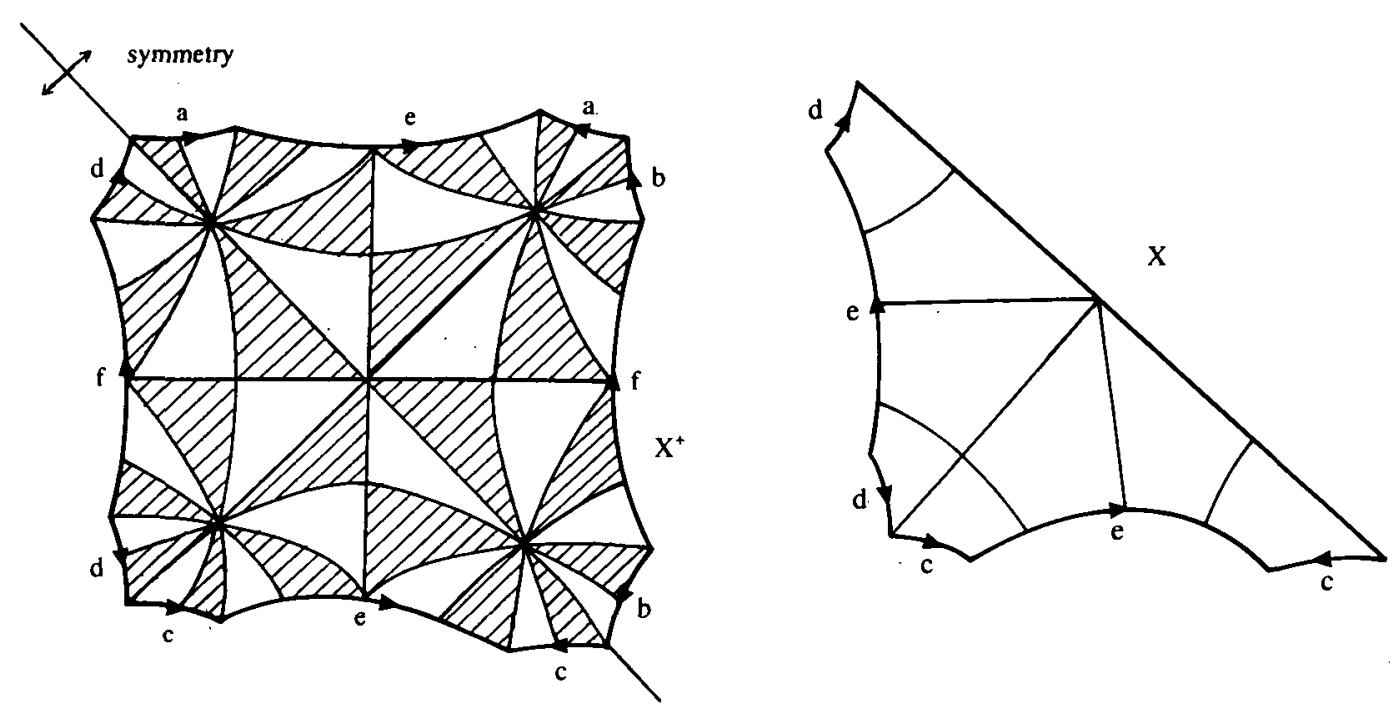

Figure 12.

previous case it follows that this exceptional surface exist and is the underlying surface of a unique regular map of type $\{4,6\}$ (see Figure 12).

Case (3). Let $\tilde{c}_{0}, \tilde{c}_{1}, \tilde{c}_{2}$ be a set of canonical generators for $\tilde{\Lambda}$. Then arguing as before (see Figure 13) one can show that

$$
c_{0}=\tilde{c}_{2} \tilde{c}_{0} \tilde{c}_{2}, \quad c_{1}=\tilde{c}_{2} \tilde{c}_{1} \tilde{c}_{2}, \quad c_{2}=\tilde{c}_{1}, \quad c_{3}=\tilde{c}_{0}
$$

is a set of canonical generators for $\Lambda$.

As before $c_{1} \in \Gamma$ or $c_{2} \in \Gamma$ and respectively $\left(c_{1} c_{3}\right)^{2} \in \Gamma^{+}$or $\left(c_{0} c_{2}\right)^{2} \in \Gamma^{+}$, which in both cases give

$$
\left(\tilde{c}_{2} \tilde{c}_{1} \tilde{c}_{2} \tilde{c}_{0}\right)^{2} \in \Gamma^{+} .
$$

Now as $\Gamma^{+}$is a Fuchsian surface group, the images $x, y$ and $z$ of $\tilde{c}_{0}, \tilde{c}_{1}$ and $\tilde{c}_{2}$ in $\tilde{G}$ are

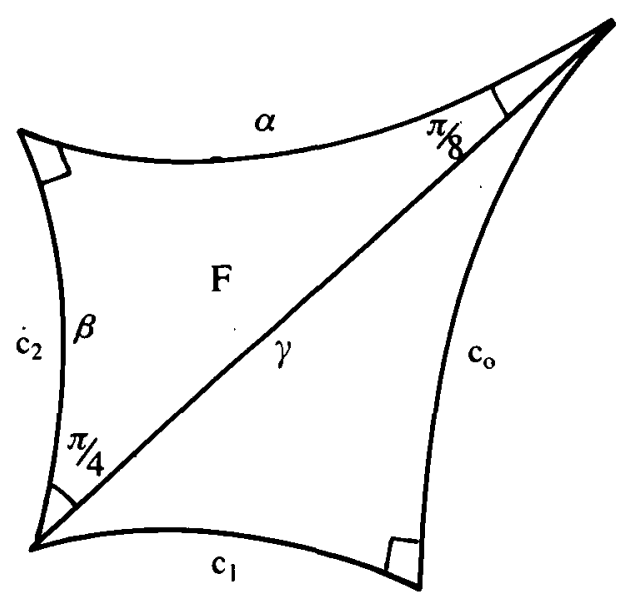

Figure 13. 
generators of order 2 such that $x y, y z$ and $x z$ have orders 2,4 and 8 respectively. Moreover by (6.10) we have $(z y z x)^{2}=1$ which is equivalent to $x(y z)^{2} x=(y z)^{2}$ and therefore $\tilde{\Lambda} / \Gamma^{+}=\tilde{G}$ is a factor group of the group with the presentation

$$
\left\langle x, y, z \mid x^{2}, y^{2}, z^{2},(x y)^{2},(y z)^{4},(x z)^{8},\left(x(y z)^{2}\right)^{2}\right\rangle .
$$

But is it clear that such group has order 64 (we remark that $(y z)^{2}$ is central) and therefore $\tilde{G}$ has order 32 or 64 . We claim that the former case is impossible. Indeed if $|\tilde{G}|=32$ then $\tilde{G}$ is a proper factor of (6.11). Clearly the images $\tilde{x}, \tilde{y}$ and $\tilde{z}$ in $\tilde{G}$ still remain elements of order 2 and $\tilde{x} \tilde{y}, \tilde{y} z$ and $\tilde{x} z$ still have orders 2,4 and 8 . So $\tilde{x}=(\tilde{y} z)^{2}$ and therefore $|\tilde{G}| \leq 8$, a contradiction.

By (2.3) $X=\mathbb{C}^{+} / \Gamma$ has algebraic genus 3. Let $c_{1} \in \Gamma$ (resp. $c_{2} \in \Gamma$ ). Then $c_{0} c_{2} \notin \Gamma$ (resp. $c_{1} c_{3} \notin \Gamma$ ) since otherwise $|\Lambda / \Gamma| \leq 4$. On the other hand $\left(c_{1} c_{3}\right)^{2},\left(c_{0}, c_{2}\right)^{2} \in \Gamma$. So $c_{1} c_{3}$ and $c_{0} c_{2}$ represent in $\Lambda / \Gamma$ elements of order 2 and therefore in both cases $\Gamma$ has four empty period cycles by [3]. So $X$ is a sphere with four holes. Observe that the above surface is hyperelliptic and the hyperelliptic involution is $\left(\tilde{c}_{1} \tilde{c}_{2}\right)^{2}$.

As before we argue that the exceptional surface $X$ exists. The surface $X$ is constructed by gluing together two isometric regular right-angled octagons in a similar way that in Section 3 to obtain the regular pair of pants. The surface $X^{+}$is the Accola-Maclachlan surface of genus 3 (see [9]) and $X^{+}$is given by the algebraic curve $y^{2}=x^{8}-1$.

Case (4). Let $\tilde{c}_{0}, \tilde{c}_{1}, \tilde{c}_{2}$ be a set of canonical generators for $\tilde{\Lambda}$. Then from Figure 14 we have that:

$$
c_{0}=\tilde{c}_{2}, \quad c_{1}=\tilde{c}_{0} \tilde{c}_{2} \tilde{c}_{0} \tilde{c}_{2} \tilde{c}_{1} \tilde{c}_{2} \tilde{c}_{0} \tilde{c}_{2} \tilde{c}_{0}, \quad c_{2}=\tilde{c}_{0} \tilde{c}_{2} \tilde{c}_{1} \tilde{c}_{2} \tilde{c}_{0}, \quad c_{3}=\tilde{c}_{1}
$$

Now $c_{1} \in \Gamma$ or $c_{2} \in \Gamma$. In the first case we obtain as $\tilde{G}$ a group of order 320 with presentation

$$
\left\langle x, y, z \mid x^{2}, y^{2}, z^{2},(x y)^{2},(y z)^{4},(x z)^{5},\left((x z)^{2} y(z x)^{2} y\right)^{2}\right\rangle .
$$

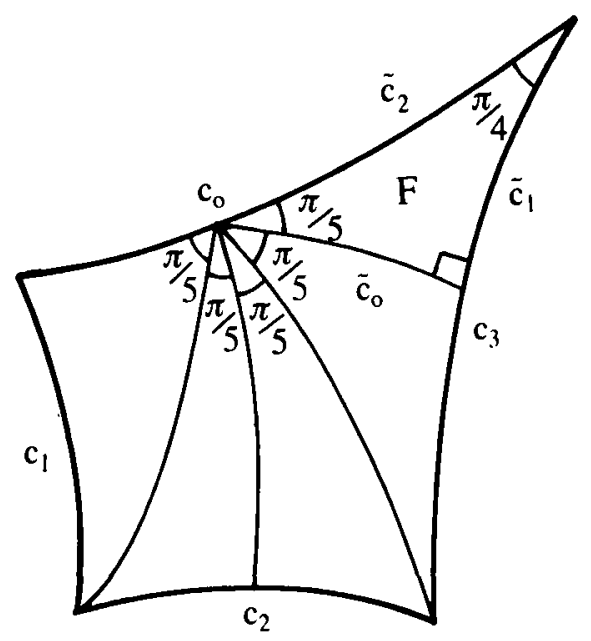

Figure 14. 

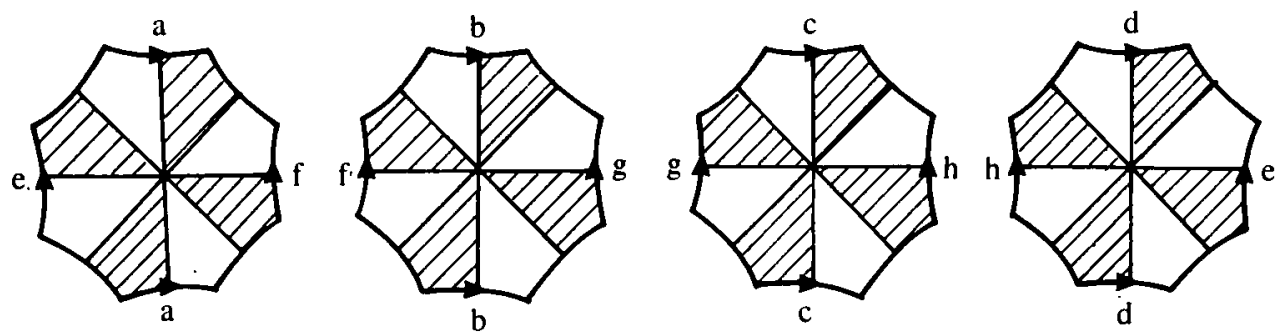

Figure 15.

If $c_{2} \in \Gamma$ we obtain a presentation for $\tilde{G}$ that tells us that $|\tilde{G}|=20$ which is impossible (see [5]).

Thus $c_{1} \in \Gamma$. In this case $\theta\left(c_{0} c_{2}\right)$ has order 4 and then $X$ must have $32 / 2 \cdot 4=4$ holes. Finally the order of $\left\langle z,(x z)^{2} y(z x)^{2}, x z y z x, y\right\rangle$ is 64 and the order of $\langle z, x z y z x, y\rangle$ is 32, then by [3]. Thm 2.1.3 the surface $X$ is orientable. The fact that $|\tilde{G}|=320$ tell us that $p=5$ and then $X$ has the topological type of a torus with four holes. The epimorphism $\theta$ give us the method of construction of $X$ and so the existence of such a surface. We remark that this surface $X$ is not hyperelliptic. If it were then $(\tilde{G})^{+}$of order 160 would have as a homomorphic image a group $H$ of automorphisms of the sphere of order 80 . We would have $H$ isomorphic to $D_{40}$ or $C_{80}$ and this is not possible. In Figure 15 we have the surface $X$ decomposed in trirectangles, using Figure 14 and doing the complex double of $X, X^{+}$, we obtain a type $\{4,5\}$ regular map on a surface of genus 5 .

7. Some results on real algebraic curves. Finally by a well known equivalence [1] between the categories of Klein surfaces and algebraic curves the results of the paper have also an interesting interpretation in terms of algebraic geometry. Namely, with only a finite number of exceptions, a real algebraic curve $C$ having a large number of birational automorphisms over $\mathbb{C}$ has a small number of such automorphisms over $\mathbb{R}$. For instance, by Theorems $\mathrm{A}$ and $\mathrm{B}$, we have:

Corollary 6.2. Let $C$ be a real algebraic curve of genus $g \geq 2$. Suppose that:

(i) the complexification of $C$ has a group of automorphisms with order greater than $24(g-1)$.

(ii) the Klein surface determined by $C$ is not any one of the six exceptions given by Theorems $A$ and $B$.

Then $\mid$ Aut $(C) \mid<8(g-1)$.

\section{REFERENCES}

1. N. L. Alling and N. Greenleaf, Foundations of the theory of Klein surfaces, Lecture Notes in Mathematics No. 219 (Springer-Verlag, 1971).

2. A. F. Beardon, The geometry of discrete groups, GTM 91, (Springer-Verlag, 1983).

3. E. Bujalance, Etayo, E., J. M. Gamboa and G. Gromadzki, Automorphism groups of compact bordered Klein surfaces, Lecture Notes in Mathematics No. 1439, (Springer-Verlag, 1990).

4. E. Bujalance, and D. Singerman, The symmetry type of a Rieman surface, Proc. London Math. Soc. (3) 51 (1985), 501-519. 
5. H. S. M. Coxeter and W. O. J. Moser, Generators and relations for discrete groups, (Springer-Verlag, 1957).

6. A. H. M. Hoare, Subgroups of NEC groups and finite permutations groups, Quart. J. Math. Oxford, Ser. 241 (1990), 45-59.

7. G. A. Jones and D. Singerman, Theory of maps on orientable surfaces, Proc. London Math. Soc. (3) 37 (1978), 273-307.

8. C. L. May, Complex doubles of bordered Klein surfaces with maximal symmetry, Glasgow Math. J. 33 (1991), 61-71.

9. D. Singerman, Symmetries and pseudo-symmetries of hyperelliptic surfaces, Glasgow Math. J., 21 (1980), 39-49.

10. W., Thurston, The geometry and topology of 3-manifolds, (Princeton University Press, to appear).

Emilio Bujalance and Antonio F. Costa

Departamento de Matemáticas Fundamentales

FACUlTad de Ciencias, UNED

MADRID 28040, SPAIN
Grzegorz Gromadzki,

Institute of Mathematics, WSP, CHOdKIEWICZA 30, 85-064

Bydgoszcz, Poland

David Singerman,

Faculty of Mathematical Studies

THE UNIVERSITY

SOUTHAMPTON SO9 5NH, UK 